\title{
Mechanism of Anti-Cancer Therapy Alopecia: An Achievement
}

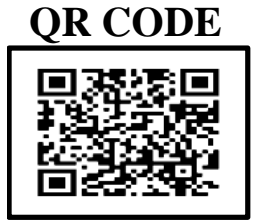

Anticancer drugs, primarily taxanes are known to induce a very harrowing and sometimes long-running hair loss also known as taxane-induced alopecia. It is a transitory and usually reversible after effect of anticancer therapy that can have a devastating effect on psychological wellbeing of the patient. ${ }^{1}$ The severe emotional trauma due to hair loss may lead to refusal or delay in cancer treatment. ${ }^{2-3}$ The resolving time may last for several months to years. Long lasting nature of this condition may result in the amplification of the psychological impact of the disease and related treatment. It is also really not known that why some patients show greater hair loss as compared to others even with the same drug and the same dosage.

Currently, scalp cooling is the only preventive treatment available for tackling chemotherapy associated alopecia, but clinical efficacy of the same is yet unsatisfactory and hard to speculate, especially in cases of taxane related hair loss. ${ }^{4}$ Marks DH et al., in a systematic review, concluded that scalp cooling systems and scalp hypothermia with cold caps are reported to show satisfactory effectiveness in the treatment of hair loss caused as an adverse reaction due to taxane chemotherapy. ${ }^{5}$ A treatment approach would only show good results if the mechanism of hair loss following chemotherapy is brought to light. Purba et al, in their sensational research, explored how taxanes (docetaxel and paclitaxel) damage human scalp hair follicles in a clinically appropriate ex-vivo organ culture model. As per their results, taxane use results in alopecia through several mechanisms including induction of the massive stockpiling of phospho-histone $\mathrm{H}_{3}+$ cells in the anagen matrix of human scalp hair follicles and agglomeration of cleaved caspase- $3+$ and $\mathrm{pH}_{3}+$ cells within the stem/progenitor-rich outer root sheath.

They also stimulate micronucleation, transcriptional arrest and cell death in keratinocytes of hair matrix. Additionally, taxanes induce cell death and mitotic defects within human hair follicle $\mathrm{K}_{15}+$ epithelial stem and progenitor cell niches. ${ }^{6}$

In their research, Purba et al. revealed how taxanes damage stem, progenitor and transit amplifying cell niches in the human hair follicles in a cell cycledependent fashion and uncovered an interestingly new management tactic for chemotherapy associated alopecia, that is hair follicle protection through targeted cell cycle arrest. They also reported that palbociclib, the $\mathrm{G} 1$ arresting $\mathrm{CDK}_{4} / 6$ inhibitor antagonises the mitosis-targeting cell toxicity of taxane chemotherapy in stem cell and transit amplifying cell compartments in the human hair follicle, without promoting premature catagen or causing adverse events related to hair follicles. ${ }^{6}$

Their recently developed and clinically relevant human model of taxane associated hair follicle damage provides an invaluable preclinical research tool for developing novel strategies to manage taxane-induced hair loss and protect against human epithelial stem cell toxicity. Moreover, they have provided a proof-of-principle of a novel therapeutic approach, i.e. pharmacological G1 arrest, that could mitigate taxane-induced hair follicle damage and prevent subsequent chemotherapy-induced alopecia through the topical delivery of G1 arresting agents. ${ }^{6}$ This breakthrough discovery will further help in unlocking more mechanisms that result in taxane induced hair loss leading to development of more safe and efficacious treatment modalities.

\section{REFERENCES:}

1. Choi EK, Kim IR, Chang O, et al. Impact of chemotherapy-induced alopecia distress on body image, psychosocial well-being, and depression in breast cancer patients. Psychooncology 2014;23:1103. 2. Lemieux J, Maunsell E, Provencher L. Chemotherapy-induced alopecia and effects on quality of life among women with breast cancer: a literature review. Psychooncology 2008;17:317.

3. Hesketh PJ, Batchelor D, Golant $\mathrm{M}$, et al. Chemotherapy - Induced alopecia: psychosocial 
impact and therapeutic approaches. Support Care Cancer 2004; 12:543.

4. Rugo HS, Klein P, Melin SA, Hurvitz SA, Melisko ME, Moore A, Park G, Mitchel

J, Bågeman E, D'Agostino RB et al. Association between use of a scalp cooling device and alopecia after chemotherapy for breast cancer. JAMA 2017; 317: 606-14.
5. Marks DH, Qureshi A, Friedman A. Evaluation of Prevention Interventions for Taxane-Induced Dermatologic Adverse Events: A Systematic Review. JAMA Dermatol. 2018;154(12):1465-72. doi: 10.1001/jamadermatol.2018.3465.

6. Purba TS, Ng'andu K, Brunken L, Smart E, Mitchell E et al. CDK4/6 inhibition mitigates stem cell damage in a novel model for taxane-induced alopecia. EMBO Mol Med. 2019;11(10):e11031

Cite this article as:

Sharma V. Mechanism of Anti-Cancer Therapy Alopecia: An Achievement. Int Healthc Res J. 2019;3(9):281-282. https://doi.org/10.26440/IHRJ/0309.12002 